\title{
Prognostic Significance of Comparison of Clinical Indicators with Manifestations of Genetic Polymorphism of Glutathione-S-Transferases in Non-Small Cell Lung Cancer
}

\author{
Mikhail N. Shapetska1 ${ }^{*}$, Evelina V. Krupnova², Alena P. Mikhalenka², Natalia V. Chebotareva², \\ Anna N. Shchayuk ${ }^{2}$, Svetlana G. Pashkevich ${ }^{3}$, Alexander V. Prokhorov ${ }^{1}$ \\ ${ }^{1}$ Belarusian State Medical University, Minsk, Republic of Belarus \\ ${ }^{2}$ Institute of Genetics and Cytology of the National Academy of Sciences of Belarus, Minsk, Republic of Belarus \\ ${ }^{3}$ Institute of Physiology of the National Academy of Sciences of Belarus, Minsk, Republic of Belarus \\ Email: *shepetjko@gmail.com
}

How to cite this paper: Shapetska, M.N., Krupnova, E.V., Mikhalenka, A.P., Chebotareva, N.V., Shchayuk, A.N., Pashkevich, S.G. and Prokhorov, A.V. (2018) Prognostic Significance of Comparison of Clinical Indicators with Manifestations of Genetic Polymorphism of Glutathione-S-Transferases in Non-Small Cell Lung Cancer. Journal of Cancer Therapy, 9, 962-973. https://doi.org/10.4236/jct.2018.912080

Received: October 17, 2018

Accepted: December 8, 2018

Published: December 11, 2018

Copyright ( 92018 by authors and Scientific Research Publishing Inc. This work is licensed under the Creative Commons Attribution International License (CC BY 4.0).

http://creativecommons.org/licenses/by/4.0/

\section{c. (i) Open Access}

\begin{abstract}
The article presented the results of comparison of polymorphic variants of the genes GSTM1, GSTT1, GSTP1 and clinical manifestations of non-small cell lung carcinoma. The association of the genotype GSTT1 (del) with the risk of developing squamous cell lung cancer has been revealed $(\mathrm{OR}=2.54$ CI: $1.13-5.72, p=0.035)$. Analysis of patient survival rate $(n=173)$ in groups of various histological types of lung cancer showed that in the group of squamous cell lung cancer $(\mathrm{n}=91)$ in patients with genotype GSTT1 (del), the survival rate median was significantly higher-84 months (95\% CI 12.4 155.7) than in patients with the genotype GSTT1 (+)-36 months (95\% CI $25.2-46.8, \mathrm{p}=0.045)$. In contrast, in the adenocarcinoma group $(\mathrm{n}=82)$, the survival rate median in patients with the genotype GSTT1 (del) was 19 months. (95\% CI 6.2 - 33.5), and in patients with genotype GSTT1 (+)-67 months (95\% CI $50.1-84.0$ ), which is the basis for continuing this comparison in an additional group of testees, as the sampling did not achieve the reliability of $p=0.12$. Hypothetically, these differences may be due to differences in the gender composition of squamous cell lung cancer and adenocarcinoma and the involvement of GST enzymes in the metabolism of estrogens in adenocarcinoma in women and other hormonal background and reactivity of the male body with squamous cell carcinoma. Further research and subsequent analysis of the results will be aimed at confirming this hypothesis.
\end{abstract}




\section{Keywords}

Squamous Cell Lung Carcinoma, Adenocarcinoma, Glutathione-S-Transferase, Survival Rate

\section{Introduction}

Among the variety of oncological diseases, malignant neoplasms of lungs are attracting close attention. In the Republic of Belarus in 2016, more than 4000 new cases of lung cancer were registered, which amounted to $10.4 \%$ of the total oncological morbidity. Despite the qualitative progress of medical technologies, mortality from this disease remains at a catastrophically high level (85\% of the number of cases). The indicator of a one-year mortality rate in 2016 was $54.9 \%$ [1]. Aggressiveness of the clinical course is mainly due to the late diagnosis and high metastatic potential of the tumor.

It is known that the risk of developing non-small cell lung cancer (NSCLC) significantly increases in smokers, especially in the environment of the inhaled air contaminated with carcinogens, capable to cause serious disturbances in the human genetic apparatus. The leading causes of the formation of the disease are the impact of environmental damage factors and disturbances in the system of maintaining the stability of the cell genome [2] [3]. Due to the fact that the alveoli and airways are on the interface between the two environments-the internal environment of the body and the external one, they are constantly exposed to the adverse effects of harmful substances that pollute the atmospheric air. Effective counteraction to various xenobiotics entering the human body is carried out by a system of coordinated enzymes for biotransformation of xenobiotics (EBX). From the point of view of modern biology and medicine, it is the metabolic status of the organism that determines the risk of development, the nature of the clinical course and the effectiveness of the treatment of malignant neoplasms. From this perspective, polymorphic variants of genes for xenobiotic biotransformation enzymes, the expression of which, unlike other classes of genes, is directly regulated by environmental factors of a chemical nature are the most promising object in the study of predisposition to lung cancer.

This objectively makes the role of enzymes of biotransformation of xenobiotics in carcinogenesis important [4] [5].

Glutathione-S-transferase is a multi-gene family of enzymes that catalyze the chemical interactions of reduced glutathione (GSH) with a wide spectrum of electrophilic compounds. In addition, as proteins, GSTs are capable of modulating signaling pathways that control cell proliferation and differentiation, apoptosis, and other processes directly involved in carcinogenesis. GSTT1, GSTM1 and GSTP1 are the most important enzymes of the GST family [6] [7].

The polymorphism of the genes GSTT1 and GSTM1 is characterized by partial or total deletion in the coding area, which leads to the absence of the corres- 
ponding enzyme. The single nucleotide polymorphism in the gene GSTP1 c.313A > G (rs1695; Ile105Val) is accompanied by a decrease in the detoxification capacity of the enzyme.

It is shown that GSTs belonging to different classes may have overlapping substrate specificity. This property, along with genetic polymorphism, causes an unequal susceptibility to environmental risk factors in different people, which by-turn is the basis for differences in individual sensitivity to genotoxic agents.

In some epidemiological studies, the role of GST polymorphisms in the initiation of a predisposition to lung cancer has been demonstrated. It has been shown that carriers of genotypes GSTM1 (del) or GSTT1 (del) have a higher risk of developing lung cancer compared to carriers of at least one functional allele [8] [9] [10]. However, most of the research results are rather contradictory. In a few studies, the association between polymorphism in GST genes and the prognosis and response to chemotherapy in patients with NSCLC has been reported, and their results are unconvincing [11] [12]. The discrepancy between the data obtained can be explained by population differences in the genotypic structure, as well as by violations of the principles of patient sampling.

This paper presents the results of a study of the associations of polymorphic variants of the genes GSTM1, GSTT1, GSTP1 with demographic, clinical characteristics and prognosis in NSCLC.

\section{Material and Investigation Methods}

The study included 173 patients diagnosed with non-small cell lung cancer who were on treatment at the Minsk City Clinical Oncology Dispensary from 2005 to 2015, permanently residing on the territory of the Republic of Belarus. The analyzed group included patients only with squamous cell carcinoma and adenocarcinoma.

The histological type of cancer and the stage of the disease were established by the morphological criteria of intraoperative biopsy during surgical treatment or tumor biopsy. The international TNM/pTNM classification was used (7th edition, 2009). The histological type of cancer was established according to the international histological classification of lung tumors (2004).

The criteria for the inclusion of patients in the analysis of the study of clinical indicators are based on the observation of classic symptoms of lung cancer, the epidemiological age characteristics of the disease origin, existing associated pathology, the smoking status, clinical and special research methods, the characteristics of the primary tumor and the extent of its spread, the morphological characteristics of the tumor, signed informed consent for participation in the study. The work was carried out in accordance with the principles of voluntariness and confidentiality, on the basis of the questioning and by authority of the ethical committee of the Belarusian State Medical University for the study of tissue samples and biological fluids.

The study did not include patients with small cell lung cancer and patients 
with a primary-multiple character of the tumor process.

The isolation of total DNA from peripheral blood was performed by the method described by Matthew [13], followed by phenol-chloroform extraction and ethanol purification. Genotyping of GSTT and GSTM polymorphisms was carried out by multiplex polymerase chain reaction method (PCR), GSTP polymorphisms-by PCR method and subsequent restriction fragment length analysis (PCR-RFLP). The primers used in the work were synthesized by the Primetech (Minsk). Reagents for PCR and PCR-RFLP were used from "Thermo Fisher Scientific" (Vilnius). After PCR-RFLP, DNA samples were visualized in UV light.

The statistical analysis was carried out with the help of the following application programs GraphPad InStat Version 3.05, SPSS Statistics 17.0 and a specialized Microsoft Excel data analysis package. To verify the validity when comparing genotype frequencies in groups, there was used a standard Pearson $\chi^{2}$ criterion or Fisher exact test for small samples criterion. The relationship between the genotypes and characteristics of the disease was assessed using the odds ratio (OR) with a $95 \%$ confidence interval (95\% CI), using the codominant and dominant SNPStats models. Overall survival rate was calculated as the time from the operation to the date of death. Survival was assessed using the Kaplan-Meier method and it was compared with the help of a log-rank test. Prognostic factors were determined using the multi-factor model of Cox proportional risk. Variables with a $\mathrm{p}$ level value of less than 0.10 after single-factor analysis were introduced into a multivariate analysis using the Cox proportional hazards model. The hazard ratio (HR) and 95\% confidence interval (95\% CI) were calculated for each factor. $\mathrm{p}<0.05$ indicates a statistical significance.

\section{Results}

The average age of the patients included in the study was $62.00 \pm 0.73$ years. According to the case history data, 2/3 (64.7\%) of the patients in the experiment group were constant smokers and only $30 \%$ of the patients denied smoking or did it sporadically. Surgery in the volume of lobectomy/bilobectomy was performed in $61.1 \%$ of cases, pneumonectomy in $20.8 \%$ of cases, lung resection$6.9 \%$ of cases. The operation was limited only to lung biopsy in $4.6 \%$ of patients and in $6.1 \%$ of cases it was not performed due to low reserve rates or the spread of the tumor process. In these cases, the disease was diagnosed on the basis of excision of the tumor with tracheobronchoscopy. I - II stage of the disease was established in $61.3 \%$ of cases, III - IV stage in $38.7 \%$ of patients. Histological forms of NSCLC were distributed in equal quantities- $52.6 \%$ squamous cell carcinoma, 47.4\%-adenocarcinoma (Table 1). In this case, among patients with squamous cell lung cancer, men dominated (92.3\%), among patients in the group with adenocarcinoma men accounted for $59.8 \%$, and women for $40.2 \%$. In the analyzed group, the genotype GSTT1 (+) was observed five times more often in $139(80.4 \%)$ patients than the genotype GSTT1 (del), which occurred in 34 (19.6\%) patients. The genotypes GSTM1 (+) and GSTM1 (del) were distributed 
Table 1. Description of the group under observation. Characteristics of the observation group.

\begin{tabular}{|c|c|}
\hline Description & Patients with NSCLC n, (\%) \\
\hline \multicolumn{2}{|l|}{ Age (years): } \\
\hline Medium & $62.0 \pm 0.73$ \\
\hline \multicolumn{2}{|l|}{ Gender: } \\
\hline fenale & $40(23.1)$ \\
\hline male & $133(76.9)$ \\
\hline \multicolumn{2}{|l|}{ Smoking: } \\
\hline no & $52(30.1)$ \\
\hline yes & $112(64.7)$ \\
\hline not clear & $9(5.2)$ \\
\hline Ethnicity: & the Slavs \\
\hline \multicolumn{2}{|l|}{ Stage: } \\
\hline I & $81(46.8)$ \\
\hline II & $25(14.5)$ \\
\hline III & $54(31.2)$ \\
\hline IV & $13(7.5)$ \\
\hline \multicolumn{2}{|l|}{ Histology: } \\
\hline squamous cell carcinoma & $91(52.6)$ \\
\hline adenocarcinoma & $82(47.4)$ \\
\hline \multicolumn{2}{|l|}{ Surgery: } \\
\hline lobectomy/bilobectomy & $106(61.1)$ \\
\hline pneumonectomy & $36(20.8)$ \\
\hline lung resection & $12(6.9)$ \\
\hline biopsy & $8(4.6)$ \\
\hline no operation & $11(6.1)$ \\
\hline \multicolumn{2}{|l|}{ Genotype: } \\
\hline GSTT1 (+) & $139(80.4)$ \\
\hline GSTT1 (del) & $34(19.6)$ \\
\hline GSTM1 (+) & $79(45.7)$ \\
\hline GSTM1 (del) & $94(54.3)$ \\
\hline GSTP (AA) & $88(50.9)$ \\
\hline GSTP1 (GA) & $68(39.3)$ \\
\hline GSTP1 (GG) & $17(9.8)$ \\
\hline
\end{tabular}

almost equally by $45.7 \%$ and $54.3 \%$, respectively. The frequency of the minor genotype GSTP1 (GG) was $9.8 \%$.

At the first stage of the work, the genetic polymorphisms GSTM1, GSTT1, GSTP1 were compared to the clinical characteristics of NSCLC (Table 2 and Table 3). 
Table 2. Association between GSTM1, GSTT1 genetic polymorphism and clinical characteristics of NSCLC.

\begin{tabular}{|c|c|c|c|c|}
\hline & \multicolumn{2}{|c|}{ GSTT1 } & \multicolumn{2}{|c|}{ GSTM1 } \\
\hline & GSTT1 (+) & GSTT1 (del) & GSTM1 (+) & GSTM1 (del) \\
\hline \multicolumn{5}{|c|}{ Histology } \\
\hline squamous cell carcinoma & $67(73.3 \%)$ & $24(26.7 \%)$ & $39(42.9 \%)$ & $52(57.1 \%)$ \\
\hline adenocarcinoma & $72(87.8 \%)$ & $10(12.2 \%)$ & $40(49.8 \%)$ & $42(51.2 \%)$ \\
\hline OR (95\% CI) & 1.00 & $2.54(1.13-5.72)$ & 1.00 & $0.71(0.39-1.31)$ \\
\hline$p$-value & & 0.035 & & 0.34 \\
\hline \multicolumn{5}{|c|}{ Gender } \\
\hline male & $102(76.7 \%)$ & $31(23.3 \%)$ & $59(44.4 \%)$ & $74(55.6 \%)$ \\
\hline vemale & $37(92.5 \%)$ & $3(7.5 \%)$ & $20(50.0 \%)$ & $20(50.0 \%)$ \\
\hline OR (95\% CI) & 1.00 & $3.75(1.08-12.99)$ & 1.00 & $1.26(0.62-2.57)$ \\
\hline$p$-value & & 0.047 & & 0.64 \\
\hline \multicolumn{5}{|c|}{ Smoking status } \\
\hline yeas & $87(77.7 \%)$ & $25(22.3 \%)$ & $52(46.8 \%)$ & $59(53.2 \%)$ \\
\hline no & $44(84.6 \%)$ & $8(15.4 \%)$ & $23(44.2 \%)$ & $29(55.8 \%)$ \\
\hline OR (95\% CI) & 1.00 & $1.50(0.62-3.62)$ & 1.00 & $1.05(0.54-2.05)$ \\
\hline$p$-value & & 0.36 & & 0.98 \\
\hline \multicolumn{5}{|c|}{ Stage } \\
\hline I-II & $87(82.1 \%)$ & $19(17.9 \%)$ & $53(50.0 \%)$ & $53(50.0 \%)$ \\
\hline III-IV & $52(77.6 \%)$ & $15(22.4 \%)$ & $26(38.8 \%)$ & $41(61.2 \%)$ \\
\hline OR (95\% CI) & 1.00 & $1.43(0.66-3.09)$ & 1.00 & $0.69(0.37-1.30)$ \\
\hline$p$-value & & 0.36 & & 0.25 \\
\hline
\end{tabular}

${ }^{*} p$ value is adjusted taking into account gender, age, and smoking status.

Table 3. Association between GSTP1 genetic polymorphisms and clinical characteristics of NSCLC.

\begin{tabular}{|c|c|c|c|c|}
\hline & $\mathrm{AA}$ & GA & GG & $\mathrm{GA}+\mathrm{GG}$ \\
\hline \multicolumn{5}{|c|}{ Histology } \\
\hline squamous cell carcinoma & $47(51.6 \%)$ & $37(40.7 \%)$ & $7(7.7 \%)$ & $44(48.4 \%)$ \\
\hline adenocarcinoma & $41(50 \%)$ & $31(37.8 \%)$ & $10(12.2 \%)$ & $41(50 \%)$ \\
\hline OR (95\% CI), & 1.00 & $1.11(0.60-2.06)$ & $0.59(0.21-1.63)$ & $0.91(0.50-1.67)$ \\
\hline$p$-value & & 0.86 & 0.44 & 0.89 \\
\hline \multicolumn{5}{|c|}{ Gender } \\
\hline male & $66(49.6 \%)$ & $56(42.1 \%)$ & $11(8.3 \%)$ & $67(50.4 \%)$ \\
\hline female & $22(55 \%)$ & $12(30 \%)$ & $6(15 \%)$ & $18(45.0 \%)$ \\
\hline OR (95\% CI), & 1.00 & $1.52(0.69-3.36)$ & $0.62(0.21-1.88)$ & $1.22(0.60-2.49)$ \\
\hline$p$-value & & 0.18 & 0.25 & 0.58 \\
\hline
\end{tabular}




\section{Continued}

\begin{tabular}{ccccc}
\hline \multicolumn{5}{c}{ Smoking status } \\
no & $61(54.5 \%)$ & $44(39.3 \%)$ & $7(6.2 \%)$ & $51(45.5 \%)$ \\
yes & $22(42.3 \%)$ & $21(40.4 \%)$ & $9(17.3 \%)$ & $30(57.7 \%)$ \\
OR (95\% CI), & 1.00 & $0.70(0.31-1.55)$ & $0.28(0.08-0.94)$ & $0.61(0.31-1.19)$ \\
$p$-value & & 0.96 & 0.043 & 0.20 \\
& $50(47.2 \%)$ & $42(39.6 \%)$ & $14(13.2 \%)$ & $56(52.8 \%)$ \\
I-II & $38(56.7 \%)$ & $26(38.8 \%)$ & $3(4.5 \%)$ & $29(43.3 \%)$ \\
III-IV & 1.00 & $0.82(0.43-1.59)$ & $0.29(0.08-1.08)$ & $0.69(0.37-1.28)$ \\
OR (95\% CI), & & 0.94 & 0.053 & 0.24 \\
$p$-value & & & \\
\hline
\end{tabular}

Among the carriers of the GSTT1 (del) genotype, the quantitative ratio was in favor of squamous cell carcinoma: 23 patients had squamous cell cancer and 10 had adenocarcinoma. Tthe study reveals the association of GSTT1 (del) genotype with the risk of developing squamous cell lung cancer (OR $=2.54 \mathrm{CI}: 1.13$ $5.72, \mathrm{p}=0.035)$. When the patients were divided according to gender, it was shown that GST1 (del) genotype was significantly more frequent in men $(\mathrm{G}=3)$ $(\mathrm{OR}=3.75 \mathrm{CI}: 1.08-12.99 ; \mathrm{p}=0.047)$. Smoking patients are significantly less likely than non-smoking patients to have the genotype GSTP1 (GG) $(\mathrm{OR}=0.28$ CI: $0.08-0.94, \mathrm{p}=0.043$ ).

At the next stage of the work, the survival rate of patients was analyzed depending on the clinical indices and polymorphic variants of the GST genes.

The disease stage was the determining factor for the outcome of treatment of patients with non-small cell lung cancer. 1-year (12 months) overall survival rate with NSCLC stage I was $91 \%$, II-70\%, III-45\% and IV-14\%. Three-year survival rate with NSCLC stage I was 74\%, II-37\%, III-28\%. Five-year survival rate with NSCLC stage I was $60 \%$, II-16\%, III-14\% (Figure 1).

Cox proportional hazards analysis showed (Table 4) that patients with stage III-IV have a worse prognosis for survival than patients with stage I-II (HR = 1.60; 95\% CI: 1.30-1.95; $\mathrm{p}<0.0001$ ).

In the general group of patients, there was no reliable association between polymorphic variants of GST genes and overall survival rate. There was also no difference in the survival of patients with squamous cell lung cancer and adenocarcinoma.

Further analysis of the survival of patients in the groups of different histological types of lung cancer revealed that in the group of squamous cell lung cancer patients with the genotype GSTT1 (del) have significantly higher survival median-84 months. (95\% CI 12.4 - 155.7) than the patients with the genotype GSTT1 (+)-36 months. (95\% CI 25.2 - 46.8), $\mathrm{p}=0.045$ (Figure 2). On the contrary, in the adenocarcinoma group, the survival median in patients with GSTT1 (del) genotype was 19 months (95\% CI 6.2 to 33.5), and in patients with GSTT1 


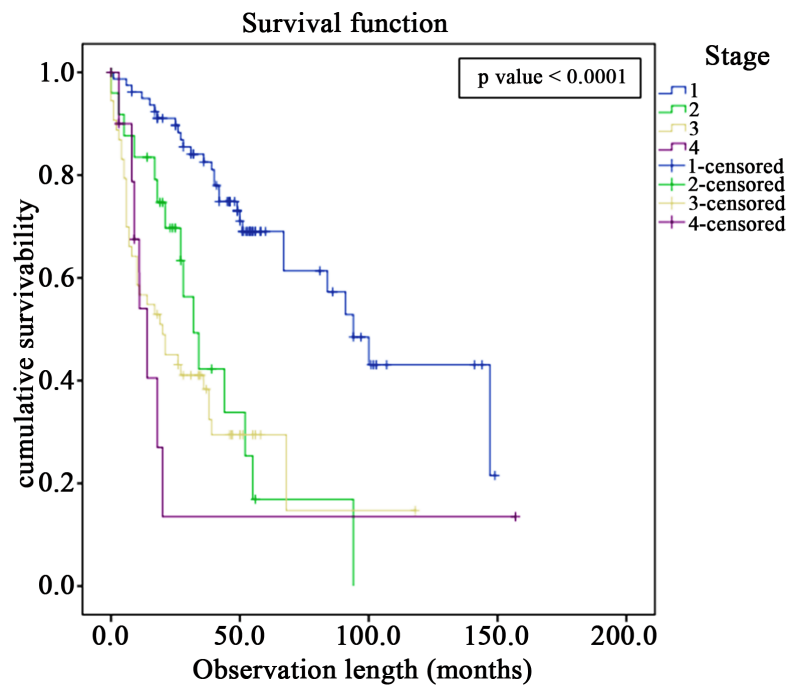

Figure 1. Kaplan-Meier survival graphs depending on the stage of the disease.

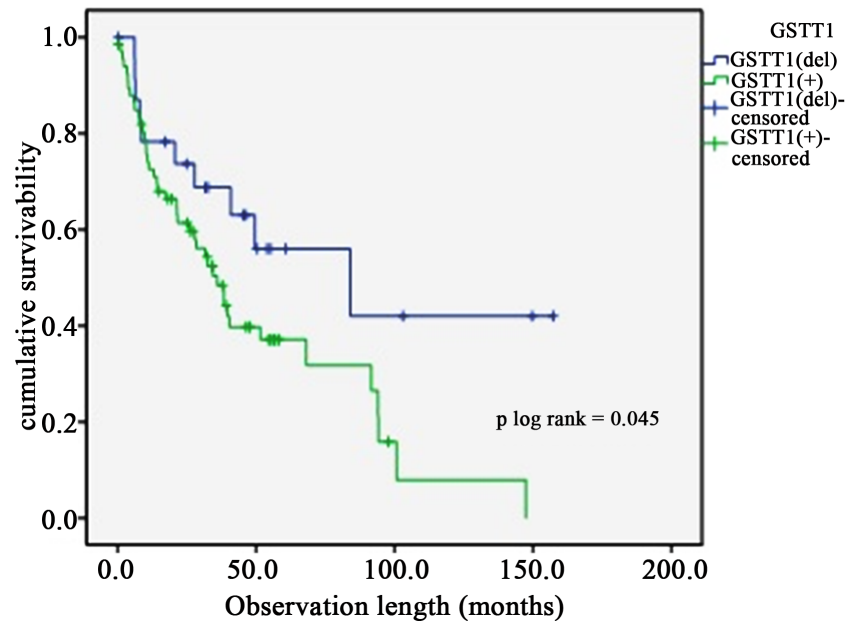

(a)

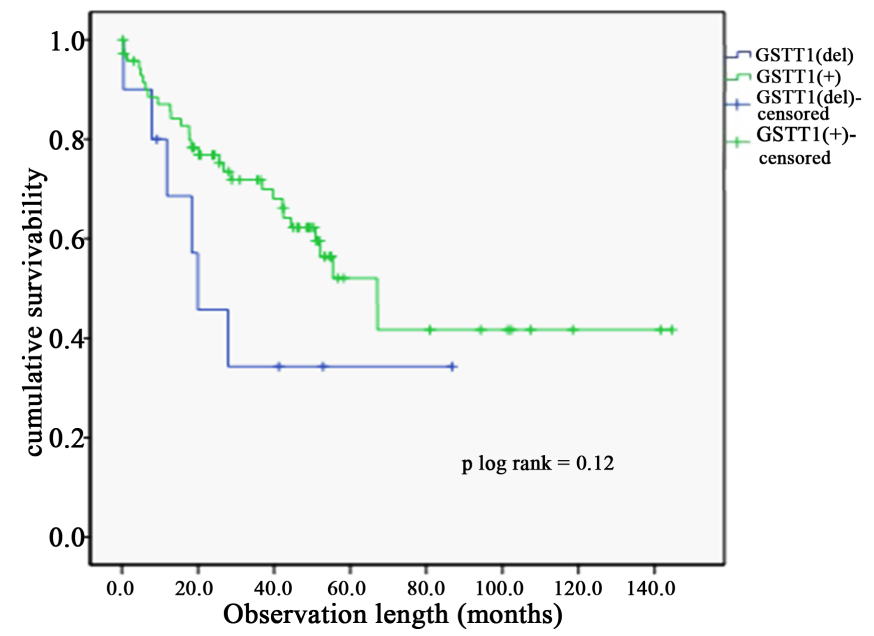

(b)

Figure 2. Kaplan-Meier graphs of survival of patients with squamous cell carcinoma (a) and adenocarcinoma (b) with GSTT1. 
Table 4. The effect of clinical characteristics and genetic polymorphism of GSTM1, GSTT1, GSTP1 on the overall survival rate of the patients with NSCLC

\begin{tabular}{|c|c|c|c|c|c|c|}
\hline & $\begin{array}{c}\text { The number } \\
\text { of dead }\end{array}$ & $\begin{array}{l}\text { The number } \\
\text { of living }\end{array}$ & $\begin{array}{l}\text { Survival rate median } \\
(95 \% \mathrm{CI}), \text { months }\end{array}$ & $p$ log-rank test & $\mathrm{HR}(95 \% \mathrm{CI})$ & $p$ value \\
\hline \multicolumn{7}{|l|}{ Stage } \\
\hline I-II & $44(41.5 \%)$ & $62(58.5 \%)$ & $84.0(45.6-122.5)$ & $<0.0001$ & 1.00 & $<0.0001$ \\
\hline III-IV & $44(65.7 \%)$ & $23(34.3 \%)$ & $18.4(9.6-27.1)$ & & $1.60(1.30-1.95)$ & \\
\hline \multicolumn{7}{|l|}{ Histology } \\
\hline Squamous cell carcinoma & $53(58.2 \%)$ & $38(41.8 \%)$ & $39.6(24.5-54.7)$ & 0.079 & 1.00 & 0.86 \\
\hline Adenocarcinoma & $35(42.7 \%)$ & $47(57.3 \%)$ & $67.1(51.4-88.8)$ & & $0.98(0.62-1.54)$ & \\
\hline \multicolumn{7}{|l|}{ gender } \\
\hline female & $9(22.5 \%)$ & $31(77.5 \%)$ & $108.5(87.2-129.8)$ & $<0.0001$ & 1.00 & 0.007 \\
\hline male & $79(59.4 \%)$ & $54(40.6 \%)$ & $39.7(29.7-49.8)$ & & $2.77(1.32-5.82)$ & \\
\hline \multicolumn{7}{|l|}{ GSTT1 } \\
\hline GSTT1 (del) & $16(47.1 \%)$ & $18(52.9 \%)$ & $49.8(0.2-105.1)$ & 0.60 & 1.00 & 0.18 \\
\hline GSTT1 (+) & $72(51.8 \%)$ & $67(48.2 \%)$ & $50.8(31.2-70.8)$ & & $0.68(0.38-1.19)$ & \\
\hline \multicolumn{7}{|l|}{ GSTM1 } \\
\hline GSTM1 (del) & $47(50.0 \%)$ & $47(50.0 \%)$ & $51.6(30.5-72.8)$ & 0.72 & 1.00 & 0.09 \\
\hline GSTM1 (+) & $41(51.9 \%)$ & $38(48.1 \%)$ & $40.9(14.9-66.9)$ & & $0.68(0.43-1.06)$ & \\
\hline \multicolumn{7}{|l|}{ GSTP1 } \\
\hline AA & $43(48.9 \%)$ & $45(51.1 \%)$ & $52.1(25.5-78.7)$ & 0.43 & 1.00 & 0.97 \\
\hline GA & $38(55.9 \%)$ & $30(44.1 \%)$ & $40.9(20.4-61.4)$ & & $1.10(0.48-2.48)$ & \\
\hline GG & $7(41.2)$ & $10(58.8)$ & $96.4(62.2-130.6)$ & & $1.01(0.48-2.50)$ & \\
\hline
\end{tabular}

(+) genotype-67 months. (95\% CI 50.1 - 84.0), but the reliability did not achieved $\mathrm{p}=0.12$, which is probably associated with a small group of carriers of GSTT1 (del) genotype.

\section{Discussion}

The study reveals a significant increase in the incidence of the patients with homozygous deletion of GSTT1 in the squamous cell carcinoma group in comparison with the patients with adenocarcinoma. A significant increase in the carriers of the GSTT1 (del) genotype among men has also been revealed. Since squamous cell carcinoma of the lung is mainly observed in men, and women are more often diagnosed with adenocarcinoma, the differences in gender groups may be related to the influence of GSTT1 (del) genotype carrier on the risk of developing squamous cell lung cancer. The study of smokers and nonsmokers groups has revealed a 2.5-fold increase in the genotype GSTP1 (GG) in a group of non-smoking patients. The main factor affecting the overall survival of patients with NSCLC is the stage of the disease: patients with I - II stages have a better prognosis, which confirms the need to search for biomarkers of early detection 
of lung cancer. Analysis of Cox proportional hazards has not revealed reliable associations between polymorphic variants of GST genes and the overall survival of patients with NSCLC. However, there is a multidirectional effect of GSTT1 genetic polymorphism on the survival of patients with different histological types of lung cancer: the survival median in carriers of GSTT1 (del) genotype with squamous cell lung cancer was significantly higher ( 84 months) than in patients with adenocarcinoma (19 months) $\mathrm{p}=0.001$ and vice versa, in the carriers of GSTT1 (+) genotype the survival median in patients with squamous cell lung cancer was significantly lower (36 months) than in patients with adenocarcinoma (67 months), $\mathrm{p}=0.03$.

Since GST enzymes are involved in the detoxification of inhaled toxic and carcinogenic electrophilic compounds, genetic polymorphisms of these enzymes are potential factors of predisposition to lung cancer [5] [14]. There is a substrate specificity of various GSTs. GSTM1 metabolizes large hydrophobic electrophiles, such as polycyclic aromatic hydrocarbon epoxides [6] [15]. GSTT1, in contrast, is involved in the metabolism of smaller compounds, such as monohalomethane and ethylene oxide [16]. GSTP1 plays an important role in the detoxification of benzapyrene diol epoxy. The catalytic activity of GST provides the cell with a mechanism to protect against harmful effects of these substances.

At present, the results of numerous studies of the role of GST gene polymorphisms in the onset of lung cancer determine GSTM1 and GSTT1 as the genes with low penetrance with an increased risk of developing lung cancer from 15\% to $21 \%$. Thus, the study by Pan et al. [17] in the Asian population has shown that the carriage of a deletion in GSTM1 or GSTT1 results in an increased risk of lung cancer with OR 1.57 (95DI 1.23 - 2.00) for GSTT1 and OR 1.87 (95\% CI 1.46 - 2.39) for GSTM1 ( $\mathrm{p}$ 0.01). The study by Pan et al. found out that the deletion of the GSTT1 gene is associated with an increased risk in men (adjusted OR $2.95,95 \%$ CI $2.07-4.20, \mathrm{p}<0.01$ ), whereas the deletion of the gene is at an increased risk among women (adjusted OR 2.95, 95\% CI $2.07-4.20, \mathrm{p}<0.01$ ). [17] So the EAGLE study conducted on the European population, has shown that a decrease in the activity of GSTM1 and GSTT1 enzymes increases the risk of lung cancer among men who smoke, and, on the contrary, is a protective factor in non-smoking women [9]. This study also shows the relationship of GSTT1 (del) genotype with an increased risk of squamous cell lung cancer. These differences are attributed to differences in the gender composition of squamous cell lung cancer (more common in male smokers) and adenocarcinoma (more common in non-smoking women).

Thus, the obtained results allow us to conclude that it is the gender differences in the histological types of NSCLC that make it possible to explain the features of the manifestation of the genetic polymorphism of GSTT1 in patients with squamous cell lung cancer and adenocarcinoma, which is important for predicting the survival of patients. The above conclusion is confirmed by the data revealing the participation of GSTM1 and GSTT1 in the metabolism of estradiol 
and in the deactivation/elimination of metabolites derived from estrogen [18] [19].

\section{Conclusion}

Thus, the conducted clinical study showed that carriers of homozygous deletion of GSTT1 are significantly more frequent among patients with squamous cell lung cancer. Multidirectional manifestations of GSTT1 polymorphism in patients with adenocarcinoma and squamous cell lung cancer have also been identified, which is associated with different patient survival patterns. The obtained data make it possible to assume that the reason for the revealed differences is the involvement of GST enzymes in the metabolism of estrogens in adenocarcinoma in women, and to assume other metabolic priorities of GST enzymes in men with squamous cell carcinoma. Further research and subsequent analysis of the results will be aimed at detailing this conclusion.

\section{Conflicts of Interest}

The authors declare no conflicts of interest regarding the publication of this paper.

\section{References}

[1] Cruz, C.S.D., Tanoue, L.T. and Matthay, R.A. (2011) Lung Cancer: Epidemiology Etiology and Prevention. Clinics in Chest Medicine, 32, 605-644. https://doi.org/10.1016/j.ccm.2011.09.001

[2] Wei, S., Wang, L.E., McHugh, M.K., Han, Y., Xiong, M., Amos, C.I., Spitz, M.R. and Wei, Q.W. (2012) Genome-Wide Gene Environment Interaction Analysis for Asbestos Exposure in Lung Cancer Susceptibility. Carcinogenesis, 33, 1531-1537. https://doi.org/10.1093/carcin/bgs188

[3] Minina, V.I., Soboleva, O.A., Glushkov, A.N., Voronina, E.N., Sokolova, E.A., Bakanova, M.L., Savchenko, Y.A., Ryzhkova, A.V., Titov, R.A., Druzhinin, V.G., Sinitsky, M.Y. and Asanov, M.A. (2017) Polymorphisms of GSTM1, GSTT1, GSTP1 Genes and Chromosomal Aberrations in Lung Cancer Patients. Journal of Cancer Research and Clinical Oncology, 143, 2235-2243. https://doi.org/10.1007/s00432-017-2486-3

[4] Frova, C. (2006) Glutathione Transferases in the Genomics Era: New Insights and Perspectives. Biomolecular Engineering, 23, 149-169.

https://doi.org/10.1016/j.bioeng.2006.05.020

[5] Langevin, S.M., Ioannidis, J.P., Vineis, P. and Taioli, E. (2010) Genetic Susceptibility to Environmental Carcinogens Group (GSEC). Assessment of Cumulative Evidence for the Association between Glutathione S-Transferase Polymorphisms and Lung Cancer: Application of the Venice Interim Guidelines. Pharmacogenet Genomics, 20, 586-597. https://doi.org/10.1097/FPC.0b013e32833c3892

[6] Hayes, J.D. and Strange, R.C. (2000) Glutathione S-Transferase Polymorphisms and Their Biological Consequences. Pharmacology, 61, 154-166.

https://doi.org/10.1159/000028396

[7] Joerger, M., Burgers, S.A., Baas, P., Smit, E.F., et al. (2012). Germline Polymorphisms in Patients with Advanced Nonsmall Cell Lung Cancer Receiving First-Line 
Platinum-Gemcitabine Chemotherapy: A Prospective Clinical Study. Cancer, 118, 2466-2475. https://doi.org/10.1002/cncr.26562

[8] Benhamou, S., Lee, W.J., Alexandrie, A.K., et al. (2002) Meta- and Pooled Analyses of the Effects of Glutathione S-Transferase M1 Polymorphisms and Smoking on Lung Cancer Risk. Carcinogenesis, 23, 1343-1350.

https://doi.org/10.1093/carcin/23.8.1343

[9] Rotunno, M., Lam, T.K., Vogt, A., Bertazzi, P.A., Lubin, J.H., Caporaso, N.E. and Landi, M.T. (2012) GSTM1 and GSTT1 Copy Numbers and mRNA Expression in Lung Cancer. Mol Carcinog, 51, E142-E150. https://doi.org/10.1002/mc.21890

[10] Pliarchopoulou, K., Voutsinas, G., Papaxoinis, G., Florou, K., Skondra, M., Kostaki, K., Roussou, P., Syrigos, K. and Pectasides, D. (2012) Correlation of CYP1A1, GSTP1 and GSTM1 Gene Polymorphisms and Lung Cancer Risk among Smokers. Oncology Letters, 3, 1301-1306. https://doi.org/10.3892/ol.2012.665

[11] Xiao, H.L., Yang, Z.T., Han, F. and Wei, H.X. (2016) Association of Glutathione S-Transferase (GST) Genetic Polymorphisms with Treatment Outcome of Cisplatin-Based Chemotherapy for Advanced Non-Small Cell Lung Cancer in a Chinese Population. Genetics and Molecular Research, 15.

https://doi.org/10.4238/gmr.15027320

[12] Ada, A.O., C Kunak, S., Hancer, F., Bilgen, S., Suzen, S.H., Alpar, S., Gulhan, M., Kurt, B. and Iscan, M. (2010) CYP and GST Polymorphisms and Survival in Advanced Non-Small Cell Lung Cancer Patients. Neoplasma, 57, 512-521. https://doi.org/10.4149/neo_2010_06_512

[13] Mathew, C.G. (1984) The Isolation of High Molecular Weight Eucaryotic DNA. Methods in Molecular Biology, 2, 31-34.

[14] Nørskov, M.S., Dahl, M. and Tybjærg-Hansen, A. (2017) Genetic Variation in GSTP1, Lung Function, Risk of Lung Cancer, and Mortality. Journal of Thoracic Oncology, 12, 1664-1672. https://doi.org/10.1016/j.jtho.2017.07.008

[15] Hayes, J.D., Flanagan, J.U. and Jowsey, I.R. (2005) Glutathione Transferases. Annual Review of Pharmacology and Toxicology, 45, 51-88. https://doi.org/10.1146/annurev.pharmtox.45.120403.095857

[16] Landi, S. (2000) Mammalian Class Theta GST and Differential Susceptibility to Carcinogens: A Review. Mutation Research, 463, 247-283. https://doi.org/10.1016/S1383-5742(00)00050-8

[17] Pan, C., Zhu, G., Yan, Z., Zhou, Y. and Liu, Z. (2014) Glutathione S-Transferase T1 and M1 Polymorphisms Are Associated with Lung Cancer Risk in a Gender-Specific Manner. Oncology Research and Treatment, 37, 164-169. https://doi.org/10.1159/000361083

[18] Raftogianis, R., Creveling, C., Weinshilboum, R. and Weisz, J. (2000) Estrogen Metabolism by Conjugation. JNCI Monographs, 27, 113-124. https://doi.org/10.1093/oxfordjournals.jncimonographs.a024234

[19] Karageorgi, S., Prescott, J., Wong, J.Y., Lee, I.M., Buring, J.E. and De Vivo, I. (2011) GSTM1 and GSTT1 Copy Number Variation in Population-Based Studies of Endometrial Cancer Risk. Cancer Epidemiology, Biomarkers \& Prevention, 20, $1447-$ 1452. https://doi.org/10.1158/1055-9965.EPI-11-0190 